\title{
Use of the colposcope in examination for sexual abuse
}

\author{
Chris Hobbs, Jane Wynne
}

\begin{abstract}
The colposcope is an instrument widely used in gynaecology for the examination of the cervix uteri. In recent years, with the recognition of the importance of child sexual abuse as a major child health problem, increased attention has focused on the examination of the genitalia and anus in children. Diagnosed cases of sexual abuse have increased substantially in recent years in both the UK and other countries. Interest in the process of diagnosis has encouraged the adoption of new methods of physical examination including the use of the colposcope. Generations of doctors were trained with little emphasis on the normal genital and anal appearances in children. Rapid growth of knowledge in this area has accumulated as a result of careful routine clinical practice and several recent research studies some of which have utilised the colposcope. ${ }^{1-3}$
\end{abstract}

\section{Medical examination in sexual abuse}

The medical examination should be seen as an important and integral part of the investigation of child abuse. In general, irrespective of the kind of abuse suspected, the child should receive a detailed medical examination the reasons for which include ${ }^{4}$ : (1) detection of any trauma or infective disorders that require treatment; (2) evaluation of the nature of any abuse; (3) provision of any forensic evidence that may be helpful to the future protection of children; (4) reassurance of the child; and (5) beginning the process of recovery.

The examination should describe the following:

- Growth and development (weigh and measure)

- Emotional state, demeanour, and behaviour during examination

- Physical state including signs of neglect

- State of clothing

- Superficial injuries, for example bruises, scratches, bites

Hospital, Leeds

General Infirmary and

Leeds University

C Hobbs

J Wynne

Correspondence to:

Dr C J Hobbs, Community

Paediatrics, St James's

University Hospital, Leeds

LS9 7TF.
- Male genitalia for bruises, abrasions, lacerations, warts, burns, etc

- Anal and perianal area for injuries, infection, dilatation, veins, fissures, scars, etc.
The child should be examined in a quiet, comfortable, and properly equipped room with toys preferably in a 'protected area' within a hospital or health centre. Difficulties can be encountered if children are examined in the middle of busy child health or hospital clinics. The examination, which is sometimes mistakenly seen as bad if not worse than the abuse, can and should be a therapeutic experience for child and family. ${ }^{5}$

The emotional impact of sexual abuse on families must not be underestimated. Because of the promiscuous nature of many sexual abusers, there are very good reasons for examining siblings of the index child and other children who may have been in contact with a suspected abuser.

\section{Description of the colposcope (fig 1)}

The essential requirements for examining the genital tract in girls include illumination and magnification. The colposcope fulfils those requirements better than any of the other methods that have been tried.

The colposcope is a binocular system of lenses of varying strength coupled to an integral light source and mounted on a rigid structure, usually a stand or cantilevered arm. Cameras ( $35 \mathrm{~mm}$, Polaroid and video) can be attached. Manufacturers of colposcopes include Olympus, Liesegang, Zeiss, Cryomedics (Cabot Medical), and Wallach.

There are variations in design and specification from one instrument to another. Models can be mounted on the examination table, or floor mounted on castor systems to enable mobility of what can be a heavy piece of equipment. Stand mounted models are lighter and therefore easier to move from one location to another. The Olympus colposcope has a cantilevered arm that can be swung to one side without moving the instrument's base. This was designed primarily for gynaecological use so that a manual pelvic examination could conveniently follow or precede colposcopy. Some instruments provide a stereoscopic image (Zeiss, Liesegang) and require an appropriate viewer to observe the photographic slides. The magnification varies from instrument to instrument but generally extends within the range of $\times 6$ to $\times 40$. Light intensity 


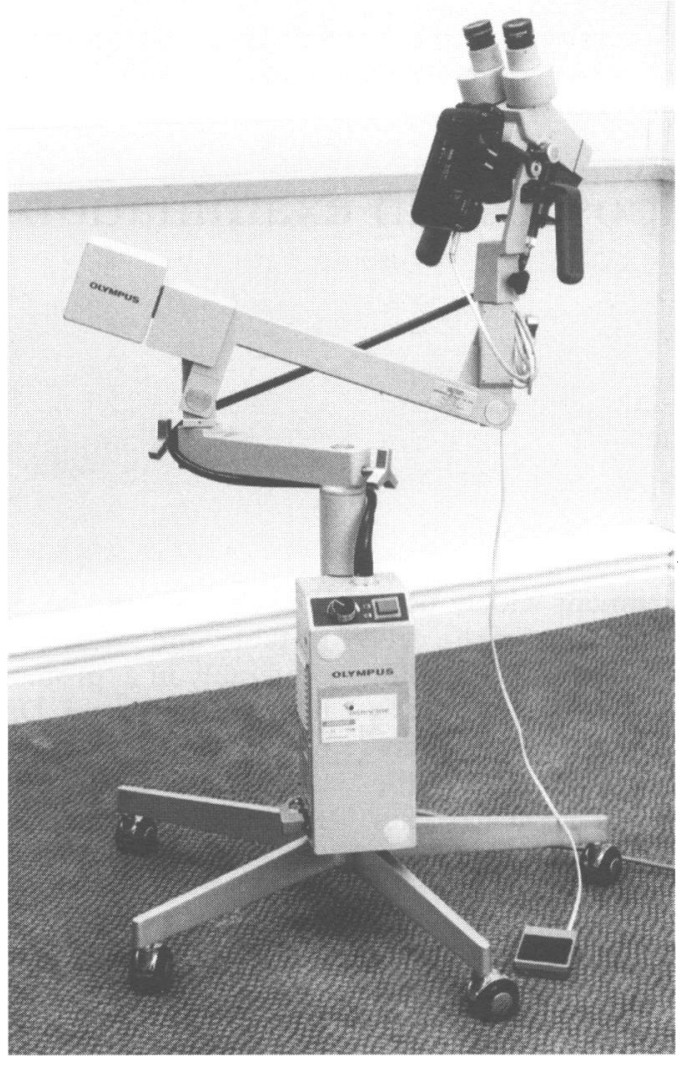

Figure 1 An Olympus colposcope. The instrument is mounted on five radiating legs fitted with castors for mobility. The light source is mounted on the central pillar and light transmitted to the head by fibreoptic cable. The binocular viewing head is mounted at the end of a counterweighted cantilever arm and is shown here with self winding $35 \mathrm{~mm}$ single lens reflex camera body attached, operated by remote foot switch. Focal distance from the front of the lens system to perineum is $30 \mathrm{~cm}$ with extension lens fitted. Variable magnification is controlled by rotating the small silver wheel above the control handle on the head (range $\times 7$ to $\times 23$ ). The arm system can be fixed by tightening the $Y$ shaped bolts.

can be varied to cope with these changes in magnification.

Most instruments incorporate a green filter to assist in the recognition of scars or abnormal vascular patterns.

\section{Use of the colposcope in sexual abuse examinations}

The first description of the colposcope's use in forensic examinations was by Teixeira in 1981 in Brazil who reported 500 patients aged 4 to 51 years. ${ }^{6} \mathrm{He}$ found that an additional $11.8 \%$ of suspected cases were corroborated than would have been using conventional examination techniques. Woodling and Heger, reporting from their experience in California, considered that an additional $10 \%$ of cases were positively identified over and above those using conventional methods. ${ }^{7}$ They commented that the colposcope becomes more important in difficult or subtle cases.

The experience of examiners in Leeds over the six or seven years since the introduction of the colposcope has been broadly similar to the Californian experience. Our ability to recognise with greater confidence both normal and abnormal findings has undoubtedly increased with this technique.
Muram and Elias reported a prospective study of 130 prepubertal girls (mean age 5.5 years) identified as victims of sexual abuse. ${ }^{8}$ The children were examined both by colposcopy and by unaided eye and it was found that of 92 girls with abnormal findings, abnormalities were observed with the unaided eye in $96 \%$. Of the four girls in whom abnormalities were initially observed only with the colposcope, on re-examination with the unaided eye the findings were found to be visible. They concluded that examination unaided was adequate for most children. Despite these contradictory views, the popularity of the colposcope continues to increase.

\section{APPLICATIONS}

The colposcope can be used in the examination of the anus in both boys and girls, and both prepubertal and pubertal genitalia in the female. Its use is non-invasive and 'creates an environment in which the examination is done with care and attention to detail'. ${ }^{7}$ The colposcope is an instrument that combines an integral photographic facility. Photography of all visible findings in abuse is increasingly expected as the standard of good practice. Images produced by colposcopy can be recorded by:

- $35 \mathrm{~mm}$ standard photography using a single lens reflex camera

- Polaroid photography producing instant photographs

- Video image - either recorded on tape, digitalised for computer analysis, converted into a photograph, or displayed on a monitor for immediate viewing, for example in physician training.

When combined with a one way screen that overlooks the consulting or examination area, physicians in training may obtain the full benefit of being able to observe the examination findings within the context of the clinical consultation with minimal intrusion for the child. Full permission including explanation of the procedure is required from the child and carers. To date, $35 \mathrm{~mm}$ photography provides the highest quality images.

An additional advantage of the colposcope is the capability, in some models with integral eye piece measuring devices, for direct measurement of examination parameters, for example the hymenal opening diameter. ${ }^{9}$ The colposcope can also facilitate the taking of fine 'ENT' sized swabs for microbiological investigation by means of transhymenal insertion under close visual control.

Why is magnification and a powerful light source important? In a prepubertal child the dimensions of the genitalia are small. The opening in the hymen is usually little more than a few $\mathrm{mm}$ in diameter and the appreciation of detail and the more subtle traumatic signs after attempted penetration will be difficult without magnification. Small areas of abrasion or petechial haemorrhage in the introitus or on the vaginal wall may be difficult if not impossible to see with the unaided eye. The detailed appearance of the hymen including the identi- 
fication of bumps, notches, and scars is clearly visualised. Clarification that unusual radial lines are anal fissures can also be assisted. Good quality photographs enable findings to be discussed with colleagues and studied with more time and attention than can usually be achieved in the emotionally charged atmosphere of the consulting room. Nowadays there is a tendency for examiners to undertake this work in teams. Each doctor has an on-call commitment and support is obtained by regular peer review meetings. The case file and photographs form the basis for discussions of cases and diagnostic evaluations can be shared with colleagues. In the USA using the new technology of videoconferencing, peer review has extended to trusted colleagues in other hospitals and centres geographically distant.

\section{ACCEPTABILITY FOR PATIENT AND PARENT}

In the modern health care system, the use of high technology is accepted and expected as an adjunct to competent and professional clinical practice. The alternative, that of attempting to peer between a child's legs with an otoscope held a short distance from the child's genitalia is less satisfactory for both examiner and patient. The colposcope places the examiner about $40 \mathrm{~cm}$ away from the child and allows a more relaxed examination. Verbal consent is usually adequate for the examination and is sought from parent and child. Additional consent is required for photographs. Information is given that photographs are used to document the medical record and may be seen by other doctors who are asked to provide opinions. If there are legal proceedings and the court permits other doctors to become involved then this information will be made available to them and may avoid the need for further undesirable examination of the child. This is especially important because most cases within the legal system will nowadays have several experts involved who usually accept good quality photographs as evidence without the need to examine the child. Use of the colposcope encourages an honest and open approach to clinical practice in a difficult area of medicine.

\section{TECHNIQUE}

The instrument can be introduced to the child so as to gain cooperation. Some children enjoy looking down it, while others find interest in the green light. The child lies in the supine position at the end of an examination couch with perineum about $25 \mathrm{~cm}$ from the end of the couch. Examination of the anus is usually undertaken with the child in the left lateral position. Ideally the colposcope should be at 90 degrees to the plane of the genitalia and anus. Our experience with the current versions of the Olympus colposcopes is that the couch may need slight elevation to achieve this with anal inspection. Modifications to the instrument are being developed. Measurements can be obtained using standard measuring devices held close to and in front of the anatomical structure to be measured and photographed. Parallax error should be avoided by close apposition.
Our current practice involves the use of an Olympus OCS3 colposcope. Olympus colposcopes account for around half of those in use in the UK at the present time. Photographs are taken with an Olympus single lens reflex camera using a Kodak Ektachrome slide film rated at $160 \mathrm{~T}$ (tungsten balanced) and without optical filtration. Film advance and correct exposure setting are performed automatically. No flash photography is required. The shutter release and film wind on are achieved by a simple foot operated switch connected via a remote cable to the camera winder. Identification of photographs is made by photographing patient details using standard information cards at the beginning of the examination. A camera data back is used to automatically imprint the date onto the photographic slide as a further check.

Many units in the USA now routinely use videocolposcopy with images stored on videotape. The full examination is then recorded.

\section{Advantages and disadvantages of the colposcope}

The advantages are:

- Non-invasive technique (colposcope is not inserted into the child)

- Good light and magnification allow detailed and careful examination

- Facilities for non-intrusive photography (requires no flash illumination). Photographs used for second opinion, legal work, teaching, and peer review ${ }^{10}$

- Acceptable to child, parents, and doctor.

The disadvantages are:

- Expensive (an basic machine will cost from around $£ 6000$ but with extra attachments could cost in excess of $£ 10000$ )

- Relatively non-portable. Earlier gynaecological instruments can be awkward to use with children

- May discourage examination in other situations by non-specialist doctors and be seen as essential

- Danger of over interpretation of minor signs.

\section{Future developments}

New digital imaging technology can permit recording of images on floppy disks and computers. Transmission via modem can facilitate immediate second opinion giving by exchange of information with remote examiners.

\section{Conclusions}

The colposcope is an acceptable non-invasive tool for examination of children where sexual abuse is suspected. Its use is gradually spreading as doctors become confident in their skills in this area. Routine recording of physical signs by means of high quality photographs should improve practice in this area and encourage examiners to agree their findings.

1 McCann J, Voris J, Simon M, Wells R. Comparison of genital examination techniques in prepubertal females. Pediatrics 1990;87:926-9. 
2 Gardner JJ. Descriptive study of genital variations in healthy, nonabused premenarchal girls. $\mathcal{F}$ Pediatr 1992;120:251-7. Hobbs CJ, Wynne JM, Thomas AJ. Colposcopic genita findings in prepubertal girls assessed for sexual abuse. Arch Dis Child 1995;73:465-9.

4 Bamford F, Roberts R. Child sexual abuse II. In: Meadow $\mathrm{R}$, ed. $A B C$ of child abuse. London: British Medical Journal, 1989:37-42.

5 De San Lazaro C. Making paediatric assessment in suspected sexual abuse a therapeutic experience. Arch Dis Child 1995;73:174-6.

6 Teixeira RG. Hymenal colposcopic examination in sexual offences. Am F Forensic Med Pathol 1981;2:209-14.
7 Woodling BA, Heger A. The use of the colposcope in the diagnosis of sexual abuse in the pediatric age group. Child Abuse Negl 1986;10:111-4.

8 Muram D, Elias S. Child sexual abuse-genital tract findings in prepubertal girls. II Comparison of colposcopic and unaided examinations. Am $\mathcal{F}$ Obstet Gynecol 1989;160: 333-5.

9 McCann J. Use of the colposcope in childhood sexual abuse examinations. Pediatr Clin North Am 1990; 37:863-80.

10 Soderstrom RM. Colposcopic documentation. An objective approach to assessing sexual abuse of girls. $\mathcal{f}$ Reprod Med 1994;39:6-8. 